Research Article

\title{
Towards Automatic Image Exposure Level Assessment
}

\author{
Lin Zhang $\left(\mathbb{D},{ }^{1}\right.$ Xilin Yang $\mathbb{D}^{1},{ }^{1}$ Lijun Zhang $\mathbb{D}^{2},{ }^{2}$ Xiao Liu $\mathbb{D}^{2},{ }^{2}$ Shengjie Zhao $\mathbb{D},^{1}$ \\ and Yong $\mathrm{Ma}$ iD $^{3}$ \\ ${ }^{1}$ School of Software Engineering, Tongji University, Shanghai, China \\ ${ }^{2}$ College of Information and Computer Sciences, University of Massachusetts Amherst, Amherst 01003, MA, USA \\ ${ }^{3}$ School of Computer Information Engineering, Jiangxi Normal University, Nanchang, China \\ Correspondence should be addressed to Lin Zhang; cslinzhang@tongji.edu.cn and Lijun Zhang; lijunzhang@cs.umass.edu
}

Received 18 July 2020; Revised 8 October 2020; Accepted 10 November 2020; Published 23 November 2020

Academic Editor: Nazrul Islam

Copyright (c) 2020 Lin Zhang et al. This is an open access article distributed under the Creative Commons Attribution License, which permits unrestricted use, distribution, and reproduction in any medium, provided the original work is properly cited.

The quality of acquired images can be surely reduced by improper exposures. Thus, in many vision-related industries, such as imaging sensor manufacturing and video surveillance, an approach that can routinely and accurately evaluate exposure levels of images is in urgent need. Taking an image as input, such a method is expected to output a scalar value, which can represent the overall perceptual exposure level of the examined image, ranging from extremely underexposed to extremely overexposed. However, studies focusing on image exposure level assessment (IELA) are quite sporadic. It should be noted that blind NR-IQA (no-reference image quality assessment) algorithms or metrics used to measure the quality of contrast-distorted images cannot be used for IELA. The root reason is that though these algorithms can quantify quality distortion of images, they do not know whether the distortion is due to underexposure or overexposure. This paper aims to resolve the issue of IELA to some extent and contributes to two aspects. Firstly, an Image Exposure Database $\left(\mathrm{IE}_{\mathrm{ps}} \mathrm{D}\right)$ is constructed to facilitate the study of $\mathrm{IELA}_{\mathrm{f}} \mathrm{IE}_{\mathrm{ps}} \mathrm{D}$ comprises 24,500 images with various exposure levels, and for each image a subjective exposure score is provided, which represents its perceptual exposure level. Secondly, as IELA can be naturally formulated as a regression problem, we thoroughly evaluate the performance of modern deep $\mathrm{CNN}$ architectures for solving this specific task. Our evaluation results can serve as a baseline when the other researchers develop even more sophisticated IELA approaches. To facilitate the other researchers to reproduce our results, we have released the dataset and the relevant source code at https://cslinzhang.github.io/imgExpo/.

\section{Introduction}

Exposure is the total amount of light falling on a photographic medium when capturing an image [1]. Improper exposure will inevitably reduce the quality of the acquired images, e.g., bringing contrast reduction. Thus, how to assess exposure levels of images (videos) and to correct ill-exposed images (videos) are of paramount importance in the research area of multimedia.

An exposure distortion is understood as the overall quality degradation caused by improper exposure. In many industrial fields, a method that can accurately assess the exposure levels of images is in urgent need [2-5]. For example, almost all the modern digital cameras can work in "autoexposure" mode [2]. When the user is taking images with this mode, the camera will automatically adjust relevant hardware parameters (such as the aperture, the shutter speed, and the electronic gain [6]) using a particular autoexposure algorithm to make the collected images have proper exposure levels. Obviously, in order to verify the performance of an autoexposure algorithm, a method that can accurately assess the exposure levels of acquired images is indispensable. Another example commonly encountered is in video surveillance. For video surveillance, it is very common that lighting conditions are out of the adaptive capacity of the camera. Hence, it is quite necessary to continuously monitor the exposure level of the acquired video to determine its quality [4].

At present, commonly adopted approaches of judging whether an image is properly exposed are based on the experience of the photographers. These kinds of schemes are of course costly and inefficient, lack robustness, and cannot be applied to systems requiring real-time 
exposure level scores. Hence, there is an urgent need to develop computational image exposure metrics.

This work tries to solve the problem of IELA (Image Exposure Level Assessment) to some extent. The ultimate goal is to obtain a computerized model that can objectively and effectively predict the overall exposure level of any given image, and the prediction results are anticipated to correlate well with human subjective judgements. The target algorithm should quantify exposure in a meaningful manner, which means that the same predicted exposure score should preferably correspond to the same exposure level across different image contents. Such an IELA algorithm has many potential applications. For example, it could be explored to measure or to optimize the performance of autoexposure models, which are of paramount importance for imaging sensor manufacturing industries.

In order to more clearly demonstrate the objectives of our work, in Figure 1, we present six images and give their exposure scores predicted by our proposed approach IEM $_{\mathrm{SN}}$ (short for "Image Exposure Metric with ShuffleNet"; refer to Section 4 for details). It should be pointed out that exposure scores predicted by IEM $_{\mathrm{SN}}$ can vary continuously from -1 to +1 . "-1" implies that the assessed image is extremely underexposed, "0" implies that it is correctly exposed, and " +1 " implies that it is extremely overexposed. The more the exposure score deviates from " 0 ," the more serious the exposure distortion is. Using $\mathrm{IEM}_{\mathrm{SN}}$, the predicted exposure scores of Figures 1(a) -1 (f) are $-0.8870,-0.5043,-0.2577$, $0.1368,0.4739$, and 0.5697 , respectively. This example demonstrates that IEM $_{\mathrm{SN}}$ 's prediction results of images' exposure levels correlate consistently with human judgements.

The rest of this article is organized as follows. Section 2 introduces the related work, our motivations, and our contributions. Section 3 presents details of $\mathrm{IE}_{\mathrm{ps}} \mathrm{D}$ (short for "Image Exposure Database"), which is our newly established benchmark dataset for the study of IELA. Section 4 presents our DCNN-based image exposure level assessment model, $I_{\mathrm{IE}}$. Experimental results and related discussions are presented in Section 5. Finally, conclusions are provided in Section 6.

\section{Related Work and Our Contributions}

In this section, we first review some representative studies most relevant to our work, including existing approaches for IELA, approaches for no-reference (NR) quality assessment of contrast-distorted images, and approaches for blind NR image quality assessment (NR-IQA). Then, our motivations and contributions are presented.

2.1. Existing Approaches for IELA. At present, the work that specializes in IELA is quite sporadic. Human experience suggests that an image's exposure level could be characterized by its luminance histogram. It is generally believed that the histogram of a correctly exposed image spreads over the whole range of luminance; by contrast, histograms of overexposed (underexposed) images are shifted to the bright (dark) sides. Moreover, the higher the exposure distortion is, the more significant will be the shift. Several IELA metrics were proposed in the literature just based on this hypothesis. In Liu et al.'s patent [7], three quantities "center," "centroid," and "effective width" are first extracted from the image's luminance histogram and then the exposure level is derived from them using predefined rules. Based on the similar idea as Liu et al.'s invention, Rychagov and Efimov [8] patented a method for exposure estimation by comparing the mean of the illuminance histogram with predefined thresholds. In Romaniak et al.'s approach $[4,9]$, the average luminance of three blocks with the highest mean luminance is regarded as the luminance upper bound $L_{U}$ and the average luminance of three blocks with the lowest mean luminance is regarded as the luminance lower bound $L_{L}$. Then, the exposure metric is calculated as $\left(L_{U}+L_{L}\right) / 2$.

\subsection{Approaches for NR Quality Assessment of Contrast-Dis-} torted Images. In most cases, improper exposure can reduce the contrast of the acquired images. Hence, studies focusing on NR quality assessment of contrast-distorted images are quite relevant to our work. The recent progress made in this area is briefly reviewed here.

On seeing that a database specially dedicated to contrastdistortion assessment was lacking, Gu et al. [10] established a database comprising contrast-changed images and their associated subjective ratings.

With respect to quality assessment models of contrastdistorted images, existing schemes can be roughly classified into two categories: the ones based on supervised learning (SL) and the ones not based on SL. Representative approaches based on SL include [11-14]. In [12], Fang et al. first derived five NSS models (in the form of probability density functions) based on the moment (mean, standard deviation, skewness, and kurtosis) and entropy features from images in SUN2012 [15]. Then, for any given image, a set of five likelihood features can be extracted based on learned NSS models. Finally, they adopted SVR (support vector regression) to find the mapping between the feature vectors and the perceptual quality scores. Inspired by Fang et al.'s idea [12], both Ahmed et al.'s work [11] and Wu et al.'s work [13] followed the similar "features + SVR" framework. In [11], Ahmed and Der extended the 5-D feature vector proposed in [12] to a 6-D one by introducing a new directional contrast feature derived from the curvelet domain. In [13], for feature extraction, $\mathrm{Wu}$ et al. extracted a 7-D feature vector (the image mean, the image variance, the image skewness, the image kurtosis, the image entropy, the mean of the phase congruency map [16], and the entropy of the phase congruency map) from each image. In $\mathrm{Xu}$ and Wang's approach [14], a 4-D feature vector, consisting of the perceptual contrast of the image, the skewness, the variance, and the intensity distribution number, is extracted from each image. Concerning the regression model mapping the feature vectors to perceptual quality scores, they resorted to a three-layer BP neural network.

Panetta et al.'s approach [17] and Gu et al.'s approach [18] are two eminent schemes for quality assessment of 


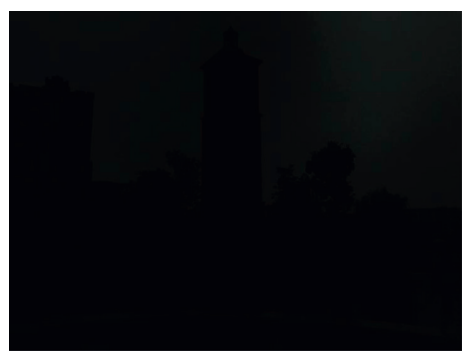

(a)

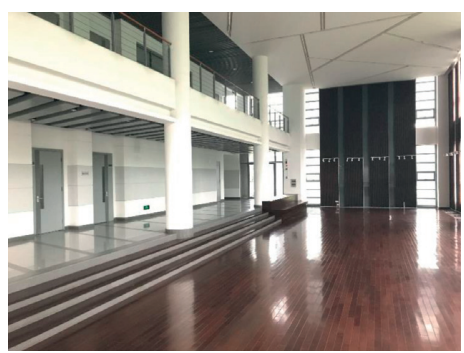

(d)

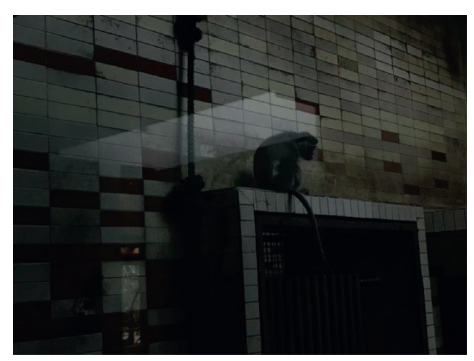

(b)

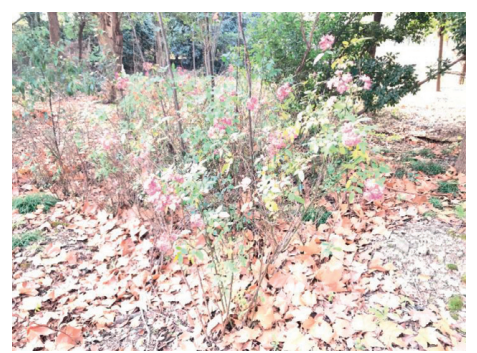

(e)

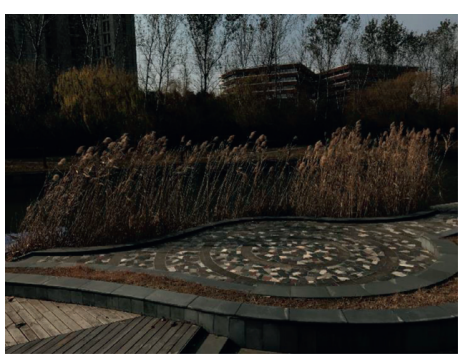

(c)

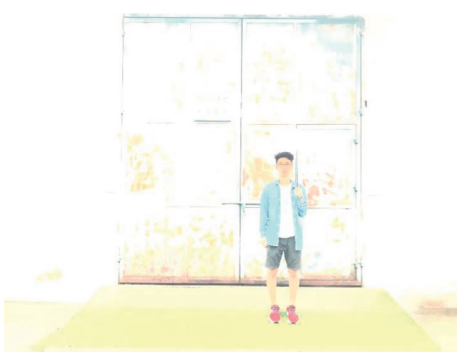

(f)

FIGURE 1: (a-f) Six images with various exposure levels. Their exposure scores predicted by our approach IEM $\mathrm{SN}_{\mathrm{S}}$ are $-0.8870,-0.5043$, $-0.2577,0.1368,0.4739$, and 0.5697 , respectively. The output range of IEM $_{\mathrm{SN}}$ is from -1 to +1 . " -1 " implies that the image is extremely underexposed, " 0 " implies that it is properly exposed, and " +1 " implies that it is extremely overexposed.

contrast-distorted images, which are not based on SL. In Panetta et al.'s approach [17], the image is first partitioned into blocks. Then, a local quality measure is derived for each block from its maximum and minimum luminance values. Finally, an overall single measure is obtained from local measures based on the PLIP (parameterized logarithmic image processing) model [19]. In [18], Gu et al. first removed predictable regions from the image and then they regarded the entropy of regions with maximum information as the local quality measure. They also derived a global quality measure by comparing the image's histogram with the uniformly distributed histogram of maximum information. Finally, an overall quality score was generated as the weighted mean of local and global measures.

2.3. Approaches for Blind NR-IQA. Another research area related to our work is blind NR-IQA, which aims to devise algorithms to predict the image's perceptual quality without knowing its high-quality reference nor its quality distortion type. Hence, the recent progress made in this area will be reviewed as well.

There exist several publicly approved databases in the area of NR-IQA. Among them, LIVE [20], CSIQ [21], LIVE Multiply Distorted (MD) [22], TID2013 [23], CID2013 [24], and LIVE Challenge [25] are most commonly used in recent studies.

With respect to blind NR-IQA models, most of the existing ones are "opinion aware," meaning that they are obtained by training on a dataset comprising quality-distorted images and the corresponding subjective scores. Typical approaches belonging to this category include [26-33], and they have similar architectures. At the training phase, the set of feature vectors is first extracted from the training images, and then a regression model that maps the feature vectors to the associated subjective scores is learned. At the testing phase, given an image $f$ to be assessed, its feature vector is extracted first and then is input into the learned regression model at the training phase to predict $f$ 's objective quality score. Different kinds of regression models are adopted in these methods, including the SVR [28, 31-33], the BP (backpropagation) neural networks [27], and the deep neural networks [26, 29, 30, 34].

Having noticed the disadvantages of opinion-aware blind NR-IQA models with respect to the generalization ability and training sample collection, some researchers proposed adding new vectorized labels to aid evaluation [35], and some researchers began to develop opinion-unaware IQA models. These kinds of models do not rely on quality-distorted training images nor subjective scores. Some eminent studies in this research direction have been reported. In [36], Mittal et al. proposed the Natural Image Quality Evaluator (NIQE) model. Given an image $f$ to be evaluated, NIQE first extracts from it a set of local features and then fits them to a multivariate Gaussian (MVG) model. The perceptual quality of $f$ is expressed as the distance between its MVG model and the MVG model learned from the image set composed of high-quality natural images. Inspired by [36], Zhang et al. [37] introduced three additional types of quality-aware features. At the test stage, on each patch of a test image, a best-fit MVG model is computed online. The overall quality score of the test image is then obtained through pooling the patch scores by averaging them. In [38], Xue et al. synthesized a virtual image set, in which the perceptual scores of the quality-distorted images were provided by FSIM (a full-reference IQA algorithm) [39]. Then, an NR-IQA model was learned from the established dataset by patch-based clustering. In [40], Wu 
et al. first extracted local features using LBP (Local Binary Pattern) [41] and then obtained statistics from local image patterns, which are believed to have the capability to discriminate high-quality natural images from distorted ones. In $\mathrm{Wu}$ et al.'s approach [42], a feature fusion scheme is first introduced by combining the image's statistical information from multiple domains and color channels. Then, the predicted image quality is generated by label transfer (LT), where a query image's KNNs (k-nearest neighbors) are searched for from some annotated images. Gu et al.'s approach [43] is based on vector regression and an objectoriented pooling strategy. By extending LBP, Freitas et al. designed the OCPP (Orthogonal Color Planes Pattern) descriptor, and they used the statistics of the OCPP descriptor to characterize image quality [44].

2.4. Our Motivations and Contributions. Through the literature survey, it can be found that though IELA is a problem of paramount importance, systematic and in-depth studies in this field are still lacking.

First, all the existing IELA metrics [4, 7-9] are derived from luminance histograms, and accordingly, their shared drawback is that they are not image content-independent. In most cases, a useful IELA metric is expected to be contentindependent. However, existing IELA metrics do not satisfy this requirement because they are totally defined on luminance histograms. As shown in Figures 2(a)-2(c), three images have the same image content, but their histograms have different distribution patterns because of their different exposure levels. The histogram of the properly exposed image (Figure 2(a)) expands over the whole luminance range, while the histogram of the overexposed (underexposed) image moves to the right (left) as shown in Figure 2(b) (Figure 2(c)). Existing IELA methods [4, 7-9] were designed precisely based on the assumption that images' perceptual exposure levels could be well characterized by their luminance histograms. However, this assumption becomes problematic when applied to images taking from various scenes. As shown in Figures 2(d)-2(f), though all three images are exposed correctly, their histogram distribution patterns differ apparently from each other owing to their different contents. As a consequence, when dealing with images similar to Figures 2(d)-2(f), IELA metrics totally based on luminance histograms [4, 7-9] would yield erroneous prediction results. In a word, the outputs of [4, 7-9] depend on image contents, and consequently, their accuracy in measuring the image exposure level is quite limited.

Second, blind NR-IQA algorithms or metrics used to measure the quality of contrast-distorted images cannot be used for IELA. When an image with improper exposure is fed into these algorithms, they can quantify its quality degradation caused by improper exposure, but the evaluation results cannot indicate whether the degradation is due to underexposure or overexposure. This fact is further illustrated by examples shown in Figure 3. By perceptual evaluation, it can be found that the images in Figures 3(a)3 (c) are underexposed, properly exposed, and overexposed, respectively. Their objective scores evaluated by "NIQMC" [10], "CS-BIQA" [33], and "IEM ${ }_{\mathrm{SN}}$ " are presented in Table 1. NIQMC is a state-of-the-art metric to measure the quality of contrast-distorted images, and a higher NIQMC score indicates higher contrast. CS-BIQA is a representative modern blind NR-IQA model, and a lower CS-BIQA score indicates higher quality. IEM $_{\mathrm{SN}}$ is our proposed IELA model (refer to Section 4 for details) trained on our established dataset used for the IELA study (refer to Section 3 for details). From Table 1, it can be seen that NIQMC and CS-BIQA can characterize an image's quality degradation quite well. However, whether the examined image is underexposed or overexposed cannot be reflected from their results. By contrast, the proposed IELA model IEM $_{\mathrm{SN}}$ can accurately and unambiguously evaluate the exposure levels of given images. The interpretation of $\operatorname{IEM}_{\mathrm{SN}}$ 's output can be found in Section 1.

Third, there is no publicly available benchmark dataset specially designed to study the IELA problem. To design and evaluate IELA approaches, such a dataset is actually indispensable.

This work attempts to fill the aforementioned research gaps partially. The major contributions are briefed as follows.

(1) To facilitate training and testing IELA models, a benchmark dataset, namely, $\mathrm{IE}_{\mathrm{ps}} \mathrm{D}$ (Image Exposure Database), has been established. $\mathrm{IE}_{\mathrm{ps}} \mathrm{D}$ contains 24,500 images with different exposure levels. 3,500 of them were collected from the real-world while the other 21,000 ones were synthesized from properly exposed source images by using our exposure simulation pipeline. For each image in $\mathrm{IE}_{\mathrm{ps}} \mathrm{D}$, a corresponding subjective score is provided to represent its perceptual exposure level. To our knowledge, $\mathrm{IE}_{\mathrm{ps}} \mathrm{D}$ is the first large-scale benchmark dataset established for the study of IELA. In our experiments, synthetic images in $\mathrm{IE}_{\mathrm{ps}} \mathrm{D}$ are used for training IELA models, while real-world ones of $\mathrm{IE}_{\mathrm{ps}} \mathrm{D}$ are used for testing. For more details about $\mathrm{IE}_{\mathrm{ps}} \mathrm{D}$, refer to Section 3 .

(2) The problem of IELA can be formulated as a regression problem from the input image to its subjective exposure score, which can be naturally solved by DCNNs (Deep Convolutional Neural Networks [45]). Hence, in this paper, a DCNN-based model IEM $_{\mathrm{X}}$ (Image Exposure Metric using $\mathrm{X}$ ) is proposed for IELA, which can learn an end-to-end mapping from images to their subjective exposure scores. Here "X" denotes a concrete DCNN architecture used. In experiments, a thorough evaluation has been conducted to assess the performance of modern DCNN architectures for IELA in the framework of IEM $_{\mathrm{X}}$ (refer to Section 5 for details).

We have released $\mathrm{IE}_{\mathrm{ps}} \mathrm{D}$ and the relevant source code at https://cslinzhang.github.io/imgExpo/ to facilitate the other researchers to reproduce our results.

A preliminary version of this manuscript has been presented on ICME 2018 [46]. The following improvements are made in this version: (1) the database $\mathrm{IE}_{\mathrm{ps}} \mathrm{D}$ is 


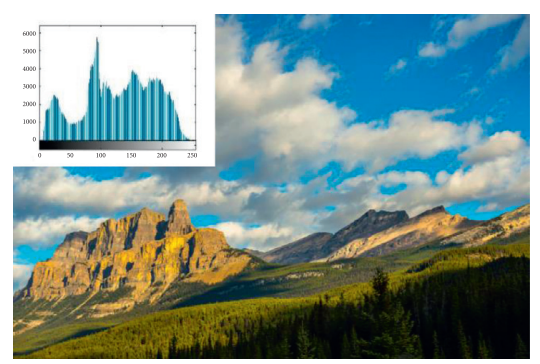

(a)

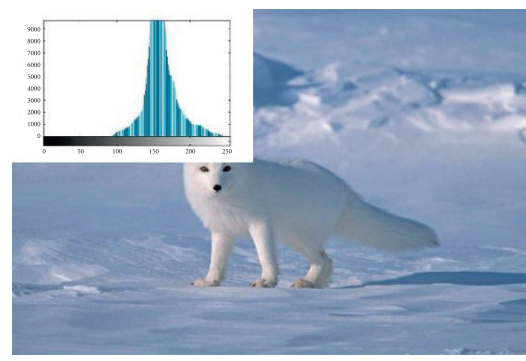

(d)

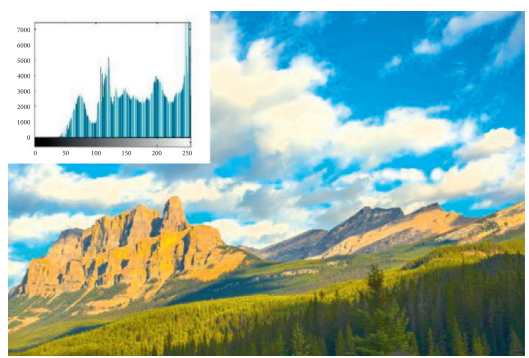

(b)

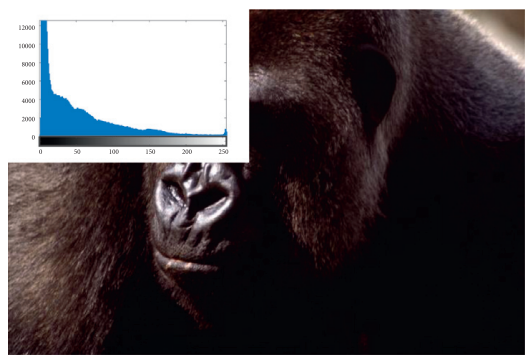

(e)

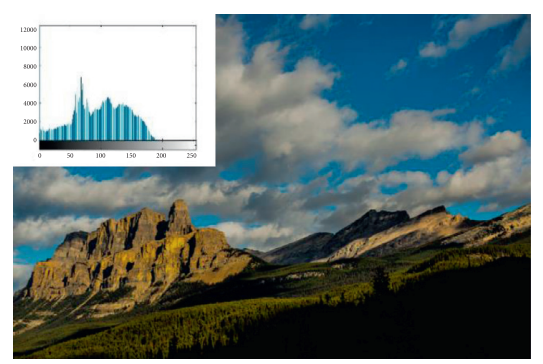

(c)

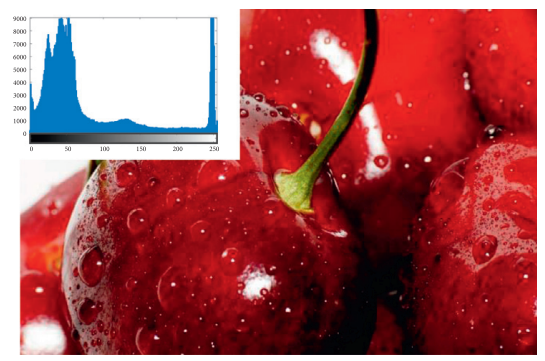

(f)

Figure 2: $(\mathrm{a}-\mathrm{c})$ Three images having the same contents but different exposure levels, along with their luminance histograms. (a) is properly exposed, while (b) and (c) are overexposed and underexposed, respectively. (d)-(f) are three images that are all properly exposed; however, their luminance histograms are quite different from each other due to their different contents.

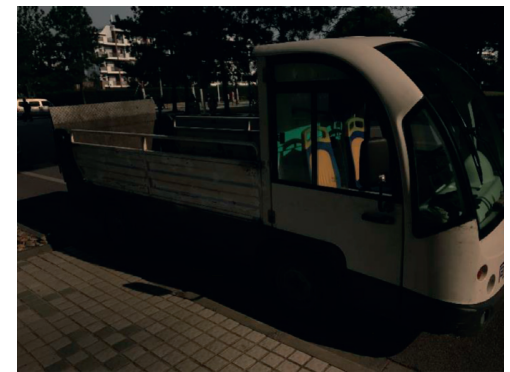

(a)

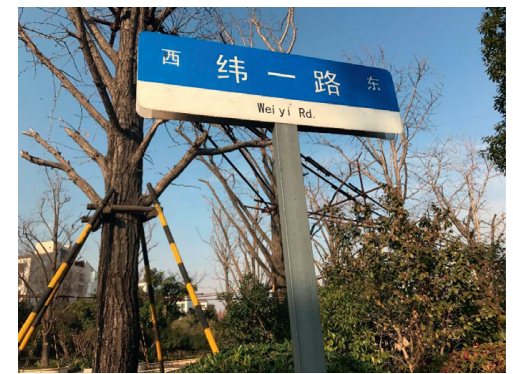

(b)

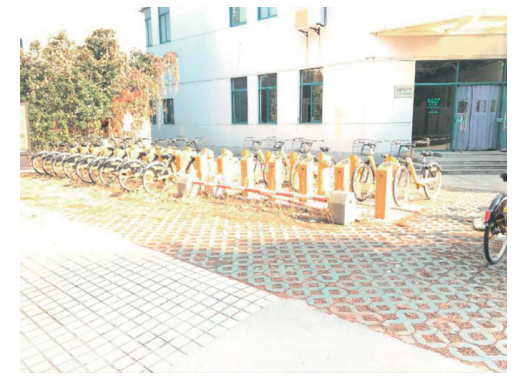

(c)

FIGURE 3: By perceptual evaluation, the images in (a), (b), and (c) are underexposed, properly exposed, and overexposed, respectively. Their objective scores predicted by different metrics are presented in Table 1.

TABle 1: Objective scores of images in Figure 3 obtained by different metrics.

\begin{tabular}{lccc}
\hline Method & Figure 3(a) & Figure 3(b) & Figure 3(c) \\
\hline NIQMC [10] & 3.9419 & 5.7086 & 3.8369 \\
CS-BIQA [33] & 3.3835 & 2.3440 & 2.7031 \\
IEM $_{S N}$ & -0.4560 & 0.0601 & 0.5697 \\
\hline
\end{tabular}

substantially extended and a more reasonable way to perform the subjective evaluation of exposure levels is adopted; (2) the performance of blind NR-IQA models and metrics used to measure the quality of contrast-distorted images for addressing the problem of IELA is thoroughly investigated and analyzed; (3) thorough performance evaluation of modern DCNN architectures in the framework of IEM $_{X}$ is conducted; and (4) more competing IELA models are evaluated in experiments.

\section{3. $\mathrm{IE}_{\mathrm{ps}} \mathrm{D}$ : A Benchmark Dataset for IELA}

As stated in Section 2, in view of the fact that a database specially dedicated to IELA still lacks in the community, we are motivated to establish such a dataset in this work. This section will discuss details about the establishment of our image exposure dataset $\mathrm{IE}_{\mathrm{ps}} \mathrm{D}$ and its practical use. By collecting and synthesizing images of various exposure levels from different shooting scenes, $\mathrm{IE}_{\mathrm{ps}} \mathrm{D}$ finally contains 24,500 images. Additionally, for each image in $\mathrm{IE}_{\mathrm{ps}} \mathrm{D}$, we provide it with a subjective score which is expected to represent its perceptual exposure level.

Three phases were involved in constructing $\mathrm{IE}_{\mathrm{ps}} \mathrm{D}$, including collection of real-world images, generation of synthetic images, and finally subjective evaluation.

3.1. Collection of Real-World Images. In order to accurately quantify an IELA algorithm's prediction accuracy on real data, $\mathrm{IE}_{\mathrm{ps}} \mathrm{D}$ should include a large number of real-world 
images. When taking these images, the shooting scenes need to be as diverse as possible, meaning that they should cover different kinds of objects (humans, plants, animals, humanmade objects, etc.), different periods of the day (morning, noon, afternoon, evening, and night), different lighting conditions, and different shooting distances. Taking these factors into consideration, we finally collected images from 500 shooting scenarios which were carefully planned. An iPhone7 Plus mobile phone was used for image collection.

For digital cameras, exposure levels can be modulated in three ways. The first way is by enlarging or shrinking the aperture. The larger the iris aperture is, the more the light reaches the imaging sensor in a fixed period of time. The second way is by adjusting the ISO sensitivity. The last way is by varying the exposure time. To simplify data collection operations, we only changed the exposure time and kept the other factors unchanged to obtain 7 different exposure results, ranging from extremely underexposed to extremely overexposed.

In the end, 3,500 $(7 \times 500)$ real-world images were collected, and we denote the dataset formed by them by $\mathrm{IE}_{\mathrm{ps}} \mathrm{D} \_\mathrm{R}$. Thumbnails of 28 sample images selected from $\mathrm{IE}_{\mathrm{ps}} \mathrm{D}$ _R are shown in Figure 4. In Figure 4, from top to bottom, images in each row belong to one specific shooting scenario; from left to right, the exposure levels are changing from "extremely overexposed" to "extremely underexposed."

\subsection{Generation of Synthetic Images with Various Exposure} Levels. To get an IELA model with a satisfying generalization capability, a large-scale dataset, comprising a large number of images with various exposure levels, is indispensable for training. Unfortunately, establishing such a real-world dataset is extremely costly and laborious. In order to resolve this contradiction, we propose to use synthetic images for training IELA models. Actually, in the community of computer vision, researchers have recently found that the use of synthetic images can effectively alleviate the problem of insufficient real training data. This has spurred the development of pipelines for synthesizing photo-realistic images. Synthetic data have already been explored to train models to tackle the problems such as object detection [47], semantic segmentation [48], optical flow estimation [49], and so on. In this paper, we propose a novel method for generating synthetic images with various exposure levels from properly exposed source images.

Suppose that $I$ is a given properly exposed source image. A synthetic image $\widehat{I}$ with a different exposure level could be created by modulating I's illumination and saturation channels. In order to manipulate the illumination and saturation channels separately, we first convert $I$ from the RGB space to the HSV space. Denote the illumination channel and the saturation channel of $I$ by $I_{v}$ and $I_{s}$, respectively. Similarly, denote the illumination channel and the saturation channel of $\widehat{I}$ by $\widehat{I}_{v}$ and $\widehat{I}_{s}$, respectively. $\widehat{I}_{v}$ is generated by adjusting $I_{v}$ as

$$
\widehat{I}_{v}(\mathbf{x})=I_{v}(\mathbf{x})+\theta \text {, }
$$

where $\mathbf{x}$ denotes the spatial location and $\theta$ is a global parameter controlling the amount of illumination adjustment. $\theta$ should be positive when simulating an overexposed image, while it should be negative when simulating an underexposed one.

In addition, $I_{s}$ needs to be adjusted to $\widehat{I}_{s}$ accordingly. As suggested by Romaniak et al. [4], the mapping function between $I(\mathbf{x})$ 's exposure level $E_{\text {in }}(\mathbf{x})$ and its saturation value $I_{s}(\mathbf{x})$ conforms to an inverse asymmetric logit function (IALF) given by the following equation:

$$
E_{\text {in }}(\mathbf{x})=\frac{\ln \left(\left(I_{s}(\mathbf{x})^{1 / c}\right) / 1-I_{s}(\mathbf{x})^{1 / c}\right)-b}{a},
$$

where $a, b$, and $c$ are three given constants. $\widehat{I}(\mathbf{x})$ 's exposure level $E_{\text {out }}(\mathbf{x})$ can be obtained by shifting $E_{\text {in }}(\mathbf{x})$ by a desired offset eps, i.e.,

$$
E_{\text {out }}(\mathbf{x})=E_{\text {in }}(\mathbf{x})+\text { eps. }
$$

At last, $\widehat{I}(\mathbf{x})$ 's saturation value $\widehat{I}_{s}(\mathbf{x})$ can be calculated by the following asymmetric logit function (ALF):

$$
\widehat{I}_{s}(\mathbf{x})=\left(\frac{e^{a * E_{\text {out }}(\mathbf{x})+b}}{1+e^{a * E_{\text {out }}(\mathbf{x})+b}}\right)^{c} .
$$

Putting equations (2)-(4) together, we can get the formula for adjusting $I_{s}$ to $\widehat{I}_{s}$ as

$$
\widehat{I}_{s}(\mathbf{x})=\left(\frac{e^{\ln I_{s}(\mathbf{x})^{1 / c}-\ln \left(1-I_{s}(\mathbf{x})^{1 / c}\right)+a * \text { eps }}}{1+e^{\ln I_{s}(\mathbf{x})^{1 / c}-\ln \left(1-I_{s}(\mathbf{x})^{1 / c}\right)+a * \text { eps }}}\right)^{c} .
$$

In our implementation, $a$ is set to -3.2 and $c$ is set to 0.4 .

By altering the values of parameters $\theta$ and eps, we can synthesize a series of I's variants with different exposure levels. Specifically, to construct $\operatorname{IE}_{\mathrm{ps}} \mathrm{D}$, seven exposure levels were synthesized. Alternatively, in other words, from each properly exposed source image, seven images (including the source image itself) having different exposure levels, ranging from "extremely underexposed" to "extremely overexposed," were synthesized. Sample synthetic images generated by our proposed scheme are shown in Figure 5. In Figure 5, images in the first column are the properly exposed source images, based on which the synthetic ones are generated. Columns 2-4 are the synthetic results of overexposed images while columns 5-7 show the synthetic results of underexposed ones. By visual inspection, it can be found that using our proposed scheme, the appearance of synthetic images looks quite natural and correlates well with human perception.

To establish the synthetic image dataset, we collected a set of properly exposed images from the Internet. Four volunteers (postgraduate students from Tongji University, Shanghai, China) were involved, and each of them was asked to search for 1000 high-quality images covering four categories: people, plants, animals, and man-made objects. Then, each of the 4000 collected images was visually examined by seven volunteer observers (undergraduate students from Tongji University). If no fewer than five of the seven observers confirmed that the image being examined was properly exposed, then the image was retained. Through this way, 3000 images were thus selected, and they were used as 


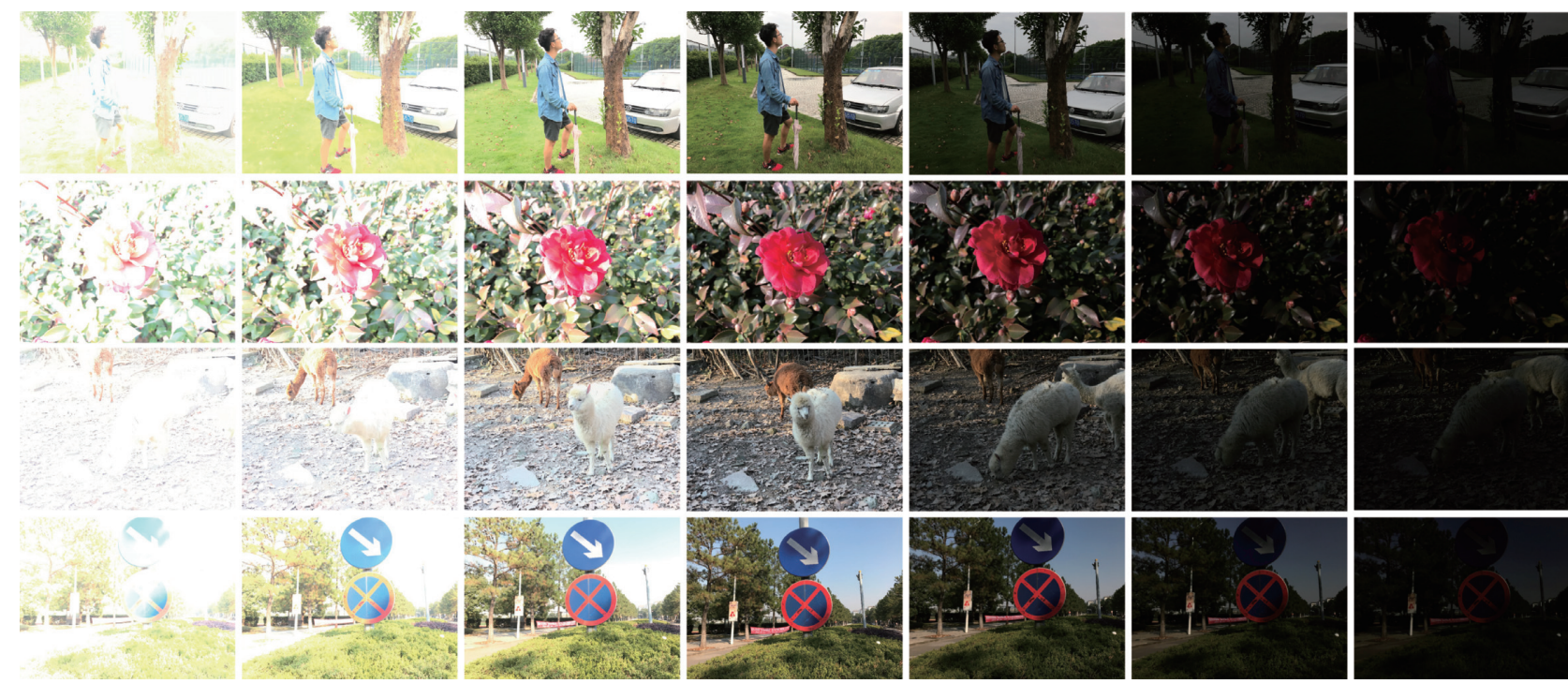

Figure 4: 28 sample images selected from $\mathrm{IE}_{\mathrm{ps}} \mathrm{D} \_\mathrm{R}$ with different exposure levels. Images in each row are taken from one specific shooting scenario. From left to right, the exposure levels are changing from "extremely overexposed" to "extremely underexposed."
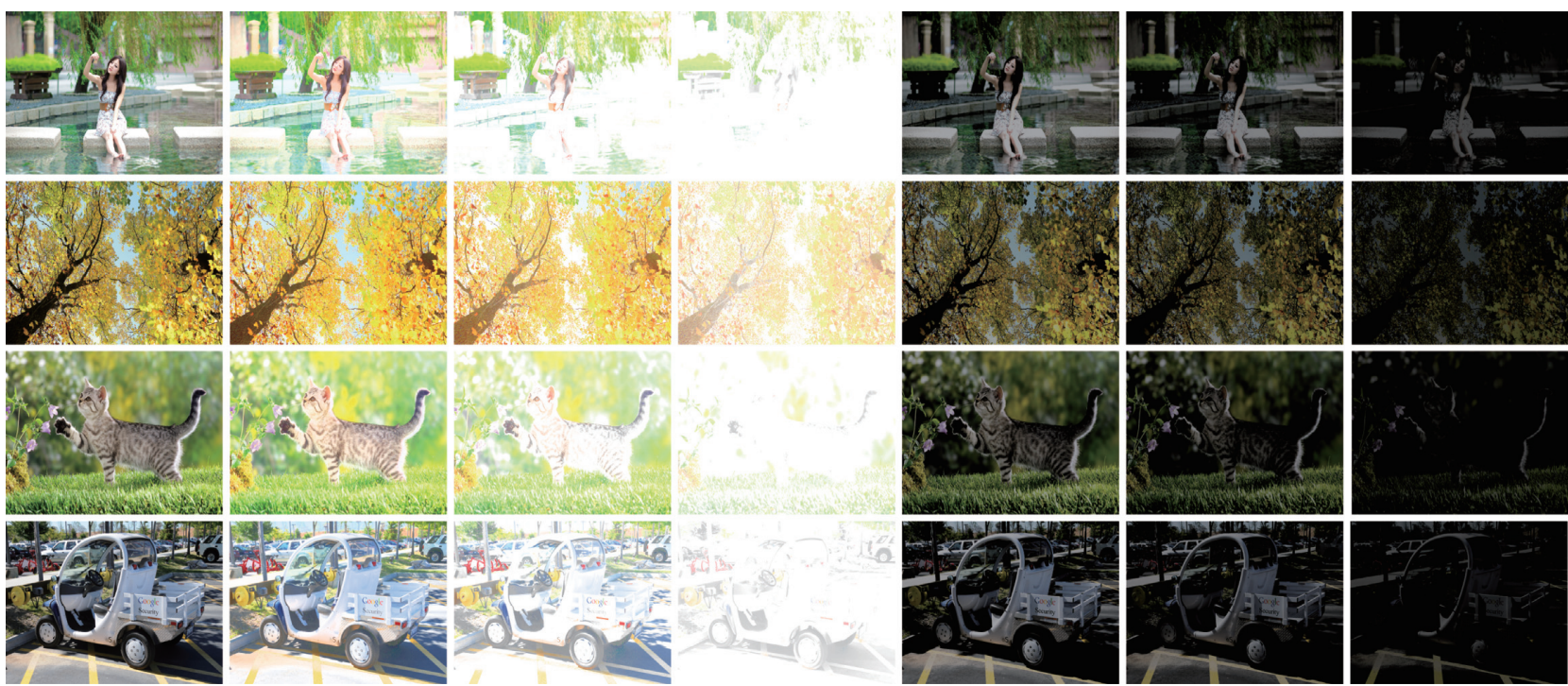

FIGURE 5: Sample synthetic images. Images in the first column are the source images. Images in columns 2-4 are synthetic overexposed images while images in columns 5-7 are synthetic underexposed ones.

source images for generating the synthetic ones. Note that none of the images used here is included in $\mathrm{IE}_{\mathrm{ps}} \mathrm{D}$ _R. Finally, using our proposed synthetic image generation model, $21,000(7 \times 3000)$ synthetic images were generated, and we denote the dataset formed by them by $\mathrm{IE}_{\mathrm{ps}} \mathrm{D} \_\mathrm{S}$.

To demonstrate the reliability of the synthetic images in the established dataset, the comparison between the real images and the generated images in terms of the brightness, which could reflect the applicability of simulated exposure levels to some extent, is conducted.

Figure 6 shows the comparison of the average brightness distributions between the real images and the synthetic images. The $X$-coordinate is the normalized average brightness value of the image. The $Y$-coordinate is the number of images. The seven colors of the bar represent different exposure levels. It can be found from the figure that the distributions of the average brightness of the real images and the synthetic images under various exposure levels are similar, which indicates that the algorithm proposed in this paper for generating synthetic images is reasonable and effective.

The final dataset $\mathrm{IE}_{\mathrm{ps}} \mathrm{D}$ comprises two parts, $\mathrm{IE}_{\mathrm{ps}} \mathrm{D} \_\mathrm{R}$ (formed by real-world images) and $\mathrm{IE}_{\mathrm{ps}} \mathrm{D} \_\mathrm{S}$ (formed by synthetic images).

3.3. Subjective Evaluation for $I E_{p s} D$. When the image set $\mathrm{IE}_{\mathrm{ps}} \mathrm{D}$ is ready, the next step is to assign a subjective score to each image in $\mathrm{IE}_{\mathrm{ps}} \mathrm{D}$, which can reflect its perceptual exposure level. 


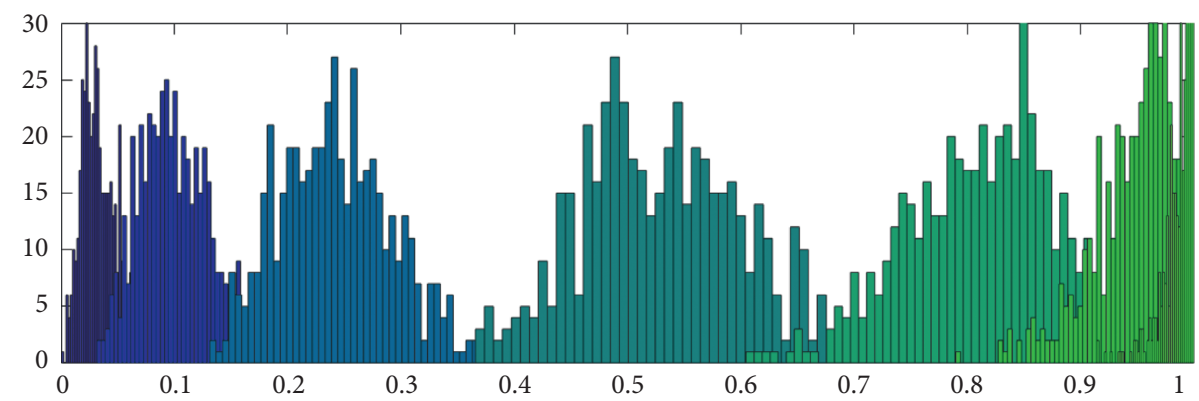

(a)

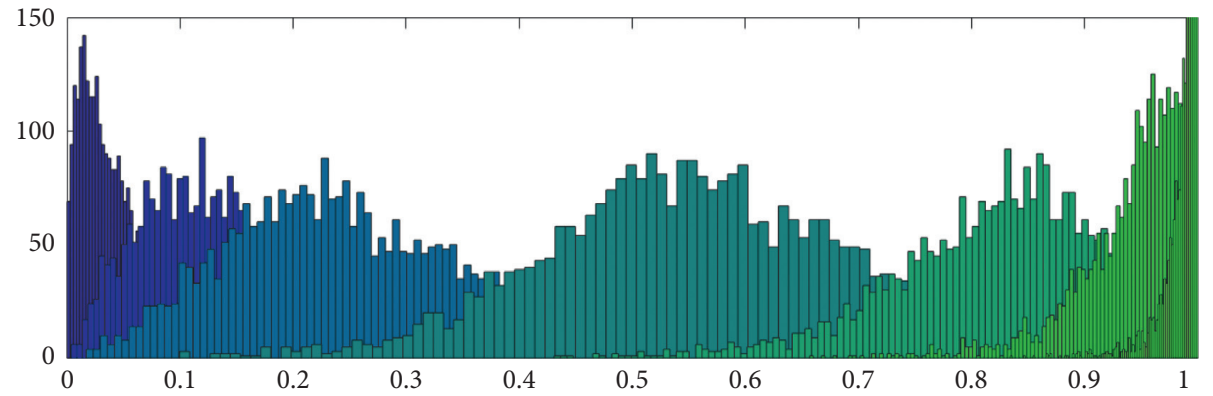

(b)

FIGURE 6: (a) The average brightness distribution of real images and (b) the average brightness distribution of synthetic images generated by our proposed algorithm.

The subjective evaluations were conducted following a single-stimulus strategy [50]. The reason for choosing a singlestimulus methodology instead of a double-stimulus one was that the number of images to be assessed was extremely huge for a double-stimulus study (we evaluated a total of 24,500 images). Subjective evaluations were performed on identical workstations. Monitors of workstations were all 22-inch LCD monitors, and their screen resolutions were all set to $1920 \times 1080$. Evaluations were conducted in an indoor environment with normal illuminations. Matlab software was developed to assist the subjective study. The lab setup is illustrated in Figure 7. Subjects taking part in the subjective evaluation were all undergraduate students of Tongji University, and they were inexperienced with image exposure level assessment. The number of subjects evaluating each image was 20 .

For each participant, we explained to him/her the goal of the experiment and also the experimental procedure. We also showed each participant the approximate range of image exposure levels and the corresponding scoring results in a short training session. It needs to be noted that we used different images in the training session from those used in the actual experiment. During the subjective evaluation, images were displayed to a subject in random order, and for different subjects, the randomization processes were different. A subject reported his/her judgement of the exposure level by dragging a slider on a quality scale. The quality scale was marked both numerically and textually and was divided into five equal portions, which were labeled as "Extremely Underexposed," "Underexposed," "Normally Exposed," "Overexposed," and "Extremely Overexposed," respectively. After the subject evaluated the image, by uniformly mapping the entire quality scale to the range $[-50,50]$, the position of the slider was converted

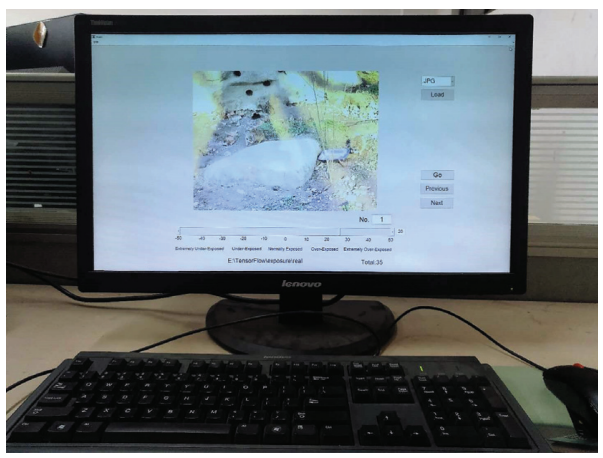

FIgURE 7: The lab setup for the subjects to evaluate the image's perceptual exposure level.

into an integer exposure score. By this way, raw exposure scores obtained from subjects were integers falling in the range $[-50$, 50]. The closer the score is to " 0 ", the more likely the image is exposed normally. A score smaller than " 0 " means the examined image is underexposed, and a score above " 0 " means the image is overexposed. Moreover, the more the exposure score deviates from "0," the more serious the exposure distortion is.

Next, some postprocessing steps were applied to subjects' raw scores. At first, to eliminate the influence of different subjective evaluation standards of subjects, the raw scores $d_{i j}$ were normalized as

$$
z_{i j}=\frac{d_{i j}-\overline{d_{i}}}{\sigma_{i}}
$$

where $d_{i j}$ is the exposure score of the image $I_{j}$ given by the $i$ th subject, $\bar{d}_{i}$ is the mean score of the $i$ th subject, $\sigma_{i}$ is the 
standard deviation of his/her scores for all images, and $z_{i j}$ is the normalized score of the image $I_{j}$ given by the $i$ th subject.

Then, we used a strategy similar to the one mentioned in [51] to filter out those heavily biased subjective scores, which satisfied

$$
z_{i j}-\overline{z_{j}}>T \cdot \sigma_{j},
$$

where $\overline{z_{j}}$ is the mean of the normalized scores of $I_{j}, T$ is a threshold constant, and $\sigma_{j}$ is the standard deviation of $I_{j}$ 's normalized scores. The mean of the remaining evaluation scores of $I_{j}$ was deemed as $I_{j}$ 's subjective exposure score $s_{j}$ :

$$
s_{j}=\frac{1}{N_{j}} \sum z_{i j}
$$

where $N_{j}$ is the number of valid subjective scores for $I_{j}$. Finally, $s_{j}$ is linearly rescaled to the range $[-1,1]$.

Now, for each image $I_{j}$ in $\mathrm{IE}_{\mathrm{ps}} \mathrm{D}$, we get a subjective score $s_{j}$ that reflects its perceptual exposure level.

3.4. Practical Use of $I E_{p s} D$. In addition to being used for IELA research, our dataset also has great potential in lots of relevant fields like high dynamic range (HDR) and image exposure correction.

HDR images can provide more dynamic range and image details and reflect the visual effects better in the real environment than ordinary images. The most common way to capture HDR images is to take a series of low dynamic range (LDR) images at different exposures and then merge them into an HDR image [52]. $\mathrm{IE}_{\mathrm{ps}} \mathrm{D}$ contains sequences of images which are very diverse and often contain complex scenes with multiple objects. Such images usually possess the same content while having different exposure levels, so they can be used to generate HDR images and to conduct related studies.

For image exposure correction, $\mathrm{IE}_{\mathrm{ps}} \mathrm{D}$ can be used as a benchmark dataset to evaluate correction methods via fullreference image quality assessment (FR-IQA) metrics. It provides properly exposed, overexposed, and underexposed images and associated subjective scores.

\section{IEMx: A DCNN-Based IELA Model}

In this section, we discuss how to build an IELA model. It is desired that such a model can accurately and efficiently predict the perceptual exposure level of a given image. Such a problem can be naturally formulated as a regression problem, which can be well addressed by DCNN (Deep Convolutional Neural Network) models [45] in an end-toend manner, mapping from input images to their associated exposure levels.

As is widely known, in the last five or six years, thanks to the emergence and maturity of DCNN, the field of multimedia processing has developed rapidly. In essence, DCNN is a representation learning technology [45]. During training, by providing a large amount of raw data to the DCNN model, it can automatically discover the suitable internal representation of data. Today, in many technical fields, DCNN-based approaches usually perform much better than
non-DCNN-based ones due to the availability of larger training sets, deeper models, better training algorithms, and more powerful GPUs. The first CNN was invented by LeCun in 1989 [53], and since the year of 2012, more elegant and powerful DCNN architectures have been continuously proposed in the literature, such as AlexNet [54], VGG [55], GoogLeNet [56], ResNet [57], DenseNet [58], and ShuffleNet [59].

We denote the proposed DCNN-based IELA model by $I_{X}$, where "IEM" is short for "Image Exposure Metric" and " $\mathrm{X}$ " represents the concrete DCNN model used (in this paper, four specific DCNN models are investigated in the framework of IEM $_{X}$, including GoogLeNet [56], ResNet [57], DenseNet [58], and ShuffleNet [59]). For training IEM $_{\mathrm{X}}$, the established dataset $\mathrm{IE}_{\mathrm{ps}} \mathrm{D} \_\mathrm{S}$ described in Section 3 is used. The lost function is defined as

$$
L(\mathbf{W})=\frac{1}{N} \sum_{j=1}^{N} \frac{1}{2}\left\|\operatorname{IEM}_{X}\left(\mathbf{W} ; I_{j}\right)-s_{j}\right\|_{2}^{2}+\frac{\lambda}{2}\|\mathbf{W}\|_{F}^{2},
$$

where $\mathbf{W}$ denotes the weights of the network, $\lambda$ is a regularization parameter, $I_{j}$ is the $j$ th training image whose subjective exposure level is $s_{j},\|\mathbf{W}\|_{F}$ returns $\mathbf{W}$ 's Frobenius norm, and $N$ is the number of training samples in $\mathrm{IE}_{\mathrm{ps}} \mathrm{D} \_\mathrm{S}$. Implementation details of IEM $_{\mathrm{X}}$ are presented in Section 5.1. The general framework of $\mathrm{IEM}_{\mathrm{X}}$ is presented in Figure 8.

\section{Experimental Results and Discussion}

5.1. Implementation Details of IEM $M_{X}$. Four state-of-the-art or representative DCNN architectures, including GoogLeNet [56], ResNet [57], DenseNet [58], and ShuffleNet [59], are investigated in the framework of IEM $_{X}$, and the corresponding concrete IELA models are referred to as IEM $_{\mathrm{GN}}$, $\mathrm{IEM}_{\mathrm{RN}}, \mathrm{IEM}_{\mathrm{DN}}$, and $\mathrm{IEM}_{\mathrm{SN}}$, respectively.

$I_{X}$ s were trained on $I_{p s} D_{-}$S. For training $\operatorname{IEM}_{X}$, we used the fine-tuning strategy, i.e., IEM $_{X}$ was fine-tuned from the deep model pretrained on ImageNet [60] for the task of image classification. Actually, for GoogLeNet, ResNet, DenseNet, and ShuffleNet, the models pretrained on ImageNet were provided by their authors and we used them directly in IEM $_{\mathrm{X}}$. TensorFlow [61] was used as our deep learning platform. Key hyperparameters used when training $I_{X} s$ were set as "optimizer" = "ADAM" [62], "learning rate" $=0.001$, "batch size" $=8$, and "weight decay" $=0.0001$.

5.2. Test Protocol. The collected dataset $\mathrm{IE}_{\mathrm{ps}} \mathrm{D} \_\mathrm{R}$ was used to evaluate the approaches' capability for predicting the image's perceptual exposure level. The performance of representative blind NR-IQA models, QA models for contrast-distorted images, and models specially designed for IELA was thoroughly studied and analyzed.

Four widely accepted metrics are adopted to evaluate the performance of the competing methods. The first two are the Spearman rank-order correlation coefficient (SROCC) and the Kendall rank-order correlation coefficient (KROCC). Both of them compute the correlation coefficients between the objective scores predicted by the IELA models and the 


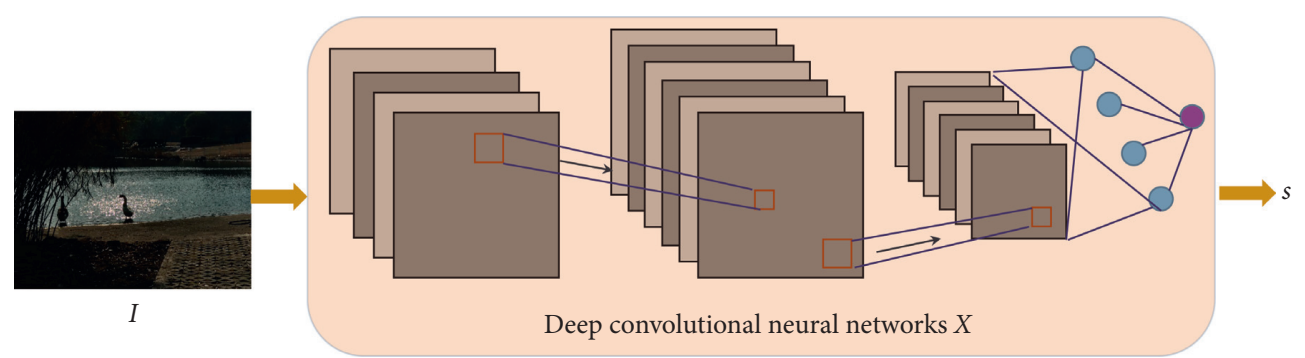

FIGURE 8: The general framework of the proposed DCNN-based IELA model IEM $\mathrm{X}_{\mathrm{X}} I$ is the input image, and $s$ is its predicted perceptual exposure level. "X" is a specific DCNN model.

subjective exposure scores provided by the dataset. SROCC is defined as

$$
\mathrm{SROCC}=1-\frac{6 \sum_{i=1}^{M} d_{i}^{2}}{M\left(M^{2}-1\right)},
$$

where $d_{i}$ is the difference between ith image's ranks in objective and subjective judgements and $M$ is the number of images in the test set. KROCC is defined as

$$
\text { KROCC }=\frac{M_{c}-M_{d}}{1 / 2 M(M-1)},
$$

where $M_{c}$ and $M_{d}$ are the numbers of concordant and discordant pairs in the test set, respectively. SROCC and KROCC are both nonparametric rank-based correlation metrics, implying that they depend only on the rank of the data points.

The third metric is the Pearson linear correlation coefficient (PLCC) between subjective scores and objective scores after a nonlinear mapping. Denote by $\left\{s_{i}\right\}_{i=1}^{M}$ and $\left\{o_{i}\right\}_{i=1}^{M}$ the set of subjective scores and the set of corresponding objective scores, respectively. First, a nonlinear mapping given by the following regression function [20] is applied to $o_{i}$ :

$$
q_{i} \equiv f\left(o_{i}\right)=\beta_{1}\left(\frac{1}{2}-\frac{1}{1+\exp \left(\beta_{2}\left(o_{i}-\beta_{3}\right)\right)}\right)+\beta_{4} o_{i}+\beta_{5},
$$

where $\beta_{i}, i=1,2, \ldots, 5$ are the model parameters that could be fitted using a nonlinear regression process to maximize the correlation between $\left\{q_{i}\right\}_{i=1}^{M}$ and $\left\{s_{i}\right\}_{i=1}^{M}$. After that, we can compute the PLCC value by

$$
\text { PLCC }=\frac{\sum_{i=1}^{M}\left(q_{i}-\bar{q}\right)\left(o_{i}-\bar{o}\right)}{\sqrt{\sum_{i=1}^{M}\left(q_{i}-\bar{q}\right)^{2}} \sqrt{\sum_{i=1}^{M}\left(o_{i}-\bar{o}\right)^{2}}} .
$$

The last metric is the RMSE (root mean squared error) between $\left\{s_{i}\right\}_{i=1}^{M}$ and $\left\{q_{i}\right\}_{i=1}^{M}$, which is defined as

$$
\mathrm{RMSE}=\sqrt{\frac{1}{M} \sum_{i=1}^{M}\left(s_{i}-q_{i}\right)^{2} .}
$$

Different from SROCC and KROCC, PLCC and RMSE can measure the prediction accuracy of IELA models.
A better IELA model is anticipated to have higher SROCC, KROCC, and PLCC values and a lower RMSE value.

\subsection{Evaluations of QA Models for Contrast-Distorted Images.} As we have stated in Section 2.2, in most cases, improper exposure can decrease the image's contrast. Thus, the studies focusing on quality assessment of contrast-distorted images (QACDI) are quite relevant to our work and it is reasonable to clearly know their performance for addressing the problem of IELA. Therefore, in this experiment, we evaluated the performance of six eminent QACDI models on $\mathrm{IE}_{\mathrm{ps}} \mathrm{D} \_\mathrm{R}$. The QACDI models evaluated included logAME [17], NR-CDIQA [12], NIQMC [18], and methods in $[11,13,14]$. It needs to be noted that NR-CDIQA and models in $[11,13,14]$ are based on supervised learning and they were trained on a subset of CSIQ [21] comprising contrast-distorted images with associated subjective quality scores.

The evaluation results are listed in Table 2. In addition, we also list the results of our IELA model IEM SN $_{\text {in Table } 2}$ for comparison.

5.4. Evaluations of Blind NR-IQA Models. Blind NR-IQA is a research area closely related to IELA. In order to clearly know whether the existing blind NR-IQA models could address the IELA problem well, in this experiment, we evaluated the performance of several prominent blind NRIQA models on $\mathrm{IE}_{\mathrm{ps}} \mathrm{D} \_\mathrm{R}$. These models include BRISQUE [31], SSEQ [28], OG-IQA [27], NOREQI [32], CS-BIQA [33], HyperIQA [63], NIQE [36], QAC [38], IL-NIQE [37], LPSI [40], and TCLT [42].

Actually, existing blind NR-IQA models can be classified into two categories, opinion-aware ones and opinion-unaware ones. The opinion-aware models are obtained by training on a dataset comprising distorted images and associated subjective scores while the opinion-unaware ones do not require those kinds of training sets. BRISQUE [31], SSEQ [28], OG-IQA [27], NOREQI [32], CS-BIQA [33], and HyperIQA [63] are opinion-aware ones, and in this experiment, we used the trained models provided by their authors (for these five blind NR-IQA schemes, models provided by the authors were all trained on the entire LIVE dataset [20]). The other five models, NIQE [36], QAC [38], 
TABLE 2: Performance evaluation of QA models for contrast-distorted images on $\mathrm{IE}_{\mathrm{ps}} \mathrm{D} \_\mathrm{R}$.

\begin{tabular}{lcccc}
\hline Methods & SROCC & KROCC & PLCC & RMSE \\
\hline logAME [17] & 0.5312 & 0.3727 & 0.6191 & 0.4256 \\
NR-CDIQA [12] & 0.2794 & 0.2367 & 0.3139 & 0.5146 \\
NIQMC [18] & -0.0399 & -0.0032 & 0.4306 & 0.4892 \\
Ahmed et al. [11] & -0.1612 & -0.0529 & 0.4730 & 0.4775 \\
Wu et al. [13] & -0.0194 & -0.0036 & 0.1091 & 0.5388 \\
Xu and Wang [14] & 0.6716 & 0.4782 & 0.7214 & 0.3753 \\
IEM $_{\text {SN }}$ & $\mathbf{0 . 9 8 5 0}$ & $\mathbf{0 . 8 8 7 6}$ & $\mathbf{0 . 9 7 5 0}$ & $\mathbf{0 . 1 0 4 7}$ \\
\hline
\end{tabular}

IL-NIQE [37], LPSI [40], and TCLT [42], are opinion-unaware ones.

Results of this experiment are reported in Table 3. In addition, we also list the results of our IELA model IEM $_{\mathrm{SN}}$ in Table 3 for comparison.

5.5. Evaluations of IELA Models. In this experiment, the performance of approaches specially designed for IELA was evaluated. The investigated approaches included Liu et al.'s approach [7], Rychagov and Efimov's approach [8], Romaniak et al.'s approach [4], IEM $\mathrm{GN}_{\mathrm{GN}}, \mathrm{IEM}_{\mathrm{RN}}, \mathrm{IEM}_{\mathrm{DN}}$, and $\mathrm{IEM}_{\mathrm{SN}}$. The latter four are the four concrete forms of our proposed IELA model IEM $_{\mathrm{X}}$. The evaluation results are reported in Table 4.

In order to make a more convincing conclusion on the performance of the models, some statistical analysis is necessary [64]. We performed a left-tailed F-test [65] based on the prediction residuals of each model. The results of the significance test are shown in Figure 9. It can be seen that our method is much better than all other models.

5.6. Discussion. Based on the experimental results reported in Sections $5.3 \sim 5.5$, the following conclusions could be drawn.

(1) Existing QACDI models or blind NR-IQA models cannot address the problem of IELA quite well. From the results presented in Tables 2 and 3, it can be seen that using these models, the assessment results of images' exposure levels do not correlate well with the subjective evaluations. Specifically, the best-performing QACDI model is $\mathrm{Xu}$ and Wang's model [14], whose SROCC value is 0.6716 , and the bestperforming blind NR-IQA model is QAC, whose SROCC value is 0.5415 . Both of them perform much worse than the approaches specially designed for IELA, whose results are reported in Table 4 .

The poor performance of blind NR-IQA algorithms and QACDI models should be mainly attributed to the fact that they cannot tell whether the quality distortion is caused by overexposure or underexposure. Another reason is that none of the existing datasets commonly used to train IQA models comprises image samples with associated subjective exposure level scores.
TABLE 3: Performance evaluation of blind NR-IQA models on $\mathrm{IE}_{\mathrm{ps}} \mathrm{D} \_\mathrm{R}$.

\begin{tabular}{lcccc}
\hline Methods & SROCC & KROCC & PLCC & RMSE \\
\hline BRISQUE & 0.1799 & 0.0887 & 0.5148 & 0.4647 \\
NIQE & -0.0566 & -0.0395 & 0.2428 & 0.5258 \\
QAC & 0.5415 & 0.3976 & 0.5960 & 0.4352 \\
IL-NIQE & 0.4399 & 0.2939 & 0.5484 & 0.4532 \\
SSEQ & 0.3565 & 0.2321 & 0.5264 & 0.4608 \\
LPSI & 0.4945 & 0.3510 & 0.5514 & 0.4521 \\
TCLT & 0.1425 & 0.0963 & 0.3081 & 0.5156 \\
OG-IQA & -0.0124 & -0.0066 & 0.1005 & 0.5393 \\
NOREQI & 0.4022 & 0.2912 & 0.4117 & 0.4938 \\
CS-BIQA & 0.4352 & 0.3147 & 0.4905 & 0.4722 \\
HyperIQA & 0.4247 & 0.3175 & 0.5132 & 0.4567 \\
IEM $_{\text {SN }}$ & $\mathbf{0 . 9 8 5 0}$ & $\mathbf{0 . 8 8 7 6}$ & $\mathbf{0 . 9 7 5 0}$ & $\mathbf{0 . 1 0 4 7}$ \\
\hline
\end{tabular}

TAble 4: Performance evaluation of IELA models on $\mathrm{IE}_{\mathrm{ps}} \mathrm{D} \_\mathrm{R}$.

\begin{tabular}{lcccc}
\hline Methods & SROCC & KROCC & PLCC & RMSE \\
\hline Liu et al. [7] & 0.7810 & 0.6860 & 0.7775 & 0.2143 \\
Rychagov and Efimov [8] & 0.8779 & 0.8360 & 0.8716 & 0.2283 \\
Romaniak et al. [4] & 0.8649 & 0.8355 & 0.86612 & 0.2496 \\
IEM $_{\mathrm{GN}}$ & 0.9827 & 0.8873 & 0.9795 & 0.1091 \\
IEM $_{\mathrm{RN}}$ & 0.9838 & 0.8867 & 0.9713 & 0.1288 \\
IEM $_{\mathrm{DN}}$ & 0.9841 & 0.8877 & 0.9774 & 0.1145 \\
IEM $_{\mathrm{SN}}$ & $\mathbf{0 . 9 8 5 0}$ & $\mathbf{0 . 8 8 7 6}$ & $\mathbf{0 . 9 7 5 0}$ & $\mathbf{0 . 1 0 4 7}$ \\
\hline
\end{tabular}

(2) The proposed DCNN-based IELA model IEM $\mathrm{X}$ performs extremely well for predicting perceptual exposure levels of real-world images. From the results listed in Table 4, it can be seen that all the variants of IEM $\mathrm{X}_{\mathrm{X}}$ can achieve high SROCC, KROCC, and PLCC values and low RMSE values. IEM ${ }_{X}$ 's performance is greatly better than the other IELA models evaluated for comparison. Especially, IEM $_{\mathrm{SN}}$ performs the best among all the models evaluated, whose SROCC and PLCC values are 0.9850 and 0.9750 , respectively.

(3) The proposed method for generating synthetic images with various exposure levels is quite reasonable. In order to provide sufficient data for training the DCNN-based IELA model IEM $_{X}$, we propose a method to generate synthetic images with various exposure levels as described in Section 3.2. With this strategy, we generated the dataset $\mathrm{IE}_{\mathrm{ps}} \mathrm{D} \_\mathrm{S}$, based on which IEM $_{X}$ was trained. Then, IEM $\mathrm{X}$ was tested on $\mathrm{IE}_{\mathrm{ps}} \mathrm{D}_{-} \mathrm{R}$, consisting of real-world images. In other words, IEM $_{\mathrm{X}}$ was trained on synthetic images, but it was tested on real-world images. The results reported in Table 4 demonstrate that even though IEM $_{X}$ s were trained on synthetic data, they perform quite well in predicting a real-world image's exposure level. This fact implies that the scheme we proposed for generating synthetic images with various exposure levels is quite effective. Such a scheme significantly reduces the cost of preparing data for training IELA models. How to effectively make use of synthetic data to solve 


\begin{tabular}{|c|c|c|c|c|}
\hline Method & Liu et al. [28] & Rychagov et al. [46] & Romaniak et al. [43] & $\mathrm{IEM}_{\mathrm{SN}}$ \\
\hline Liu et al. [28] & 0 & 1 & 0 & 0 \\
\hline Rychagov et al. [46] & 0 & 0 & 0 & 0 \\
\hline Romaniak et al. [43] & 0 & 1 & 0 & 0 \\
\hline $\mathrm{IEM}_{\mathrm{SN}}$ & 1 & 1 & 1 & 0 \\
\hline
\end{tabular}

Figure 9: The results of the significance test on dataset $\mathrm{IE}_{\mathrm{ps}} \mathrm{D} \_\mathrm{R}$. A value of 1 means that the first model (indicated by the row) has better performance than the second model (indicated by the column), with a confidence level of greater than $95 \%$. A value of 0 means that the first model is not significantly better than the second model. If the value is always 0 no matter which of the two models is the first one, there is no significant difference in the performance of the two models.

vision problems should be given more attention by researchers.

\section{Conclusion and Future Work}

IELA models are highly desired in some vision-related industries. However, systematic studies specially focusing on this issue are still lacking. This work attempts to fill this research gap, and the contributions are from two aspects. First, an Image Exposure Database, namely, $\mathrm{IE}_{\mathrm{ps}} \mathrm{D}$, containing 24,500 images with multiple exposure levels, was established. For each image in $\operatorname{IE}_{\mathrm{ps}} \mathrm{D}$, we provide it a subjective exposure score representing its perceptual exposure level. $\mathrm{IE}_{\mathrm{ps}} \mathrm{D}$ can serve as a benchmark to train and test IELA models. To the best of our knowledge, $\mathrm{IE}_{\mathrm{ps}} \mathrm{D}$ is the first of its kind. Second, we formulated the IELA problem as a regression problem and proposed a DCNN-based solution $\mathrm{IEM}_{\mathrm{X}}$. Four specific DCNN architectures, GoogLeNet, ResNet, DenseNet, and ShuffleNet, were investigated in the framework of IEM $M_{X}$. Experimental results show that IEM $_{X}$ yields much better exposure level prediction performance than all the compared competing methods. Experimental results also corroborate that blind NR-IQA models or QACDI models could not yield acceptable performance when being exploited to address the IELA issue. In near future, we will consider how to embed IELA metrics into the design of autoexposure algorithms.

\section{Data Availability}

The relevant source code and dataset have been made publicly available at https://cslinzhang.github.io/imgExpo/.

\section{Conflicts of Interest}

The authors declare no conflicts of interest.

\section{Acknowledgments}

This study was supported in part by the National Natural Science Foundation of China under grant nos. 61973235, 61936014, and 61972285, in part by the Natural Science Foundation of Shanghai under grant no. 19ZR1461300, and in part by the Shanghai Science and Technology Innovation Plan under grant no. 20510760400.

\section{References}

[1] B. London, J. Stone, and J. Upton, Photography: Essential Way, Prentice-Hall, Upper Saddle River, NJ, USA, 2007.

[2] T. Kuno, H. Sugiura, and N. Matoba, "A new automatic exposure system for digital still cameras," IEEE Transactions on Consumer Electronics, vol. 44, no. 1, pp. 192-199, 1998.

[3] S. Liu and X. Zhang, "Image decolorization combining local features and exposure features," IEEE Transactions on Multimedia, vol. 21, no. 10, pp. 2461-2472, 2019.

[4] P. Romaniak, L. Janowski, M. Leszczuk, and Z. Papir, "A no reference metric for the quality assessment of videos affected by exposure distortion," in Proceedings of the IEEE International Conference on Multimedia and Expo, pp. 1-6, IEEE, Barcelona, Spain, July 2011.

[5] T.-H. Wang, C.-W. Chiu, W.-C. Wu et al., "Pseudo-multipleexposure-based tone fusion with local region adjustment," IEEE Transactions on Multimedia, vol. 17, no. 4, pp. 470-484, 2015.

[6] J. Torres and J. M. Menéndez, "Optimal camera exposure for video surveillance systems by predictive control of shutter speed, aperture, and gain," in Real-Time Image and Video Processing, pp. 238-251, International Society for Optics and Photonics, Bellingham, WA, USA, 2015.

[7] M. Liu, P. Yuan, and R. S. Turner, "Automatic analysis and adjustment of digital images with exposure problems," US Patent 20040190789A1, 2004.

[8] M. Rychagov and S. Efimov, "Image Processing Device to Determine Image Quality and Method Thereof, and recording medium," US Patent 20050238227A1, 2007.

[9] P. Romaniak, L. Janowski, M. Leszczuk, and Z. Papir, “The design of an objective metric and construction of a prototype system for monitoring perceived quality (QoE) of video sequences," Journal of Telecommunications and Information Technology, vol. 2011, no. 3, pp. 87-94, 2011.

[10] K. Gu, G. Zhai, W. Lin, and M. Liu, "The analysis of image contrast: from quality assessment to automatic enhancement," IEEE Transactions on Cybernetics, vol. 46, no. 1, pp. 284-297, 2016.

[11] I. T. Ahmed and C. S. Der, "No-reference image quality assessment algorithm for contrast-distorted images enhanced by using directional contrast feature in curvelet domain," in Proceedings of the IEEE International Colloquium on Signal 
Processing and its Applications, pp. 61-66, Batu Ferringhi, Malaysia, March 2017.

[12] Y. Fang, K. Ma, Z. Wang, W. Lin, Z. Fang, and G. Zhai, "Noreference quality assessment of contrast-distorted images based on natural scene statistics," IEEE Signal Processing Letters, vol. 22, no. 7, pp. 838-842, 2015.

[13] J. Wu, Z. Xia, Y. Ren, and H. Li, "No-reference quality assessment for contrast-distorted image," in Proceedings of the International Conference on Image Processing Theory, Tools and Applications, IEEE, Oulu, Finland, December 2016.

[14] M. Xu and Z. Wang, "No-reference quality assessment of contrast-distorted images," in Proceedings of the International Conference on Signal and Image Processing, IEEE, Dubai, UAE, 2016.

[15] J. Xiao, J. Hays, K. A. Ehinger, A. Oliva, and A. Torralba, "Sun database: large-scale scene recognition from abbey to zoo," in Proceedings of the IEEE Computer Society Conference on Computer Vision and Pattern Recognition, pp. 3485-3492, San Francisco, CA, USA, June 2010.

[16] P. Kovesi, "Image features from phase congruency," Videre: Journal of Computer Vision Research, vol. 1, no. 3, pp. 1-26, 1999.

[17] K. A. Panetta, E. J. Wharton, and S. S. Agaian, "Human visual system-based image enhancement and logarithmic contrast measure," IEEE Transactions on Systems, Man, and Cybernetics, Part B (Cybernetics), vol. 38, no. 1, pp. 174-188, 2008.

[18] K. Gu, W. Lin, G. Zhai, X. Yang, W. Zhang, and C. W. Chen, "No-reference quality metric of contrast-distorted images based on information maximization," IEEE Transactions on Cybernetics, vol. 47, no. 12, pp. 4559-4565, 2017.

[19] K. Panetta, S. Agaian, Y. Yicong Zhou, and E. J. Wharton, "Parameterized logarithmic framework for image enhancement," IEEE Transactions on Systems, Man, and Cybernetics, Part B (Cybernetics), vol. 41, no. 2, pp. 460-473, 2011.

[20] H. R. Sheikh, M. F. Sabir, and A. C. Bovik, "A statistical evaluation of recent full reference image quality assessment algorithms," IEEE Transactions on Image Processing, vol. 15, no. 11, pp. 3440-3451, 2006.

[21] E. C. Larson and D. M. Chandler, "Most apparent distortion: full-reference image quality assessment and the role of strategy," Journal of Electronic Imaging, vol. 19, no. 1, pp. 1-21, 2010.

[22] D. Jayaraman, A. Mittal, A. K. Moorthy, and A. C. Bovik, "Objective quality assessment of multiply distorted images," in Proceedings of the 2012 Conference Record of the Forty Sixth Asilomar Conference on Signals, Systems and Computers (ASILOMAR), pp. 1693-1697, Pacific Grove, CA, USA, November 2012.

[23] N. Ponomarenko, O. Ieremeiev, V. Lukin et al., "Color image database TID2013: peculiarities and preliminary results," in Proceedings of the European Workshop on Visual Information Processing, pp. 106-111, IEEE, Paris, France, June 2013.

[24] T. Virtanen, M. Nuutinen, M. Vaahteranoksa, P. Oittinen, and J. Häkkinen, "CID2013: a database for evaluating noreference image quality assessment algorithms," IEEE Transactions on Image Processing, vol. 24, no. 1, pp. 390-402, 2015.

[25] D. Ghadiyaram and A. C. Bovik, "Massive online crowdsourced study of subjective and objective picture quality," IEEE Transactions on Image Processing, vol. 25, no. 1, pp. 372-387, 2016.

[26] S. Bosse, D. Maniry, K.-R. Müller, T. Wiegand, and W. Samek, "Deep neural networks for no-reference and full-reference image quality assessment," IEEE Transactions on Image Processing, vol. 27, no. 1, pp. 206-219, 2018.

[27] L. Liu, Y. Hua, Q. Zhao, H. Huang, and A. C. Bovik, "Blind image quality assessment by relative gradient statistics and adaboosting neural network," Signal Processing: Image Communication, vol. 40, pp. 1-15, 2016.

[28] L. Liu, B. Liu, H. Huang, and A. C. Bovik, "No-reference image quality assessment based on spatial and spectral entropies," Signal Processing: Image Communication, vol. 29, no. 8, pp. 856-863, 2014.

[29] K. Ma, W. Liu, T. Liu, Z. Wang, and D. Tao, “dipIQ: blind image quality assessment by learning-to-rank discriminable image pairs," IEEE Transactions on Image Processing, vol. 26, no. 8, pp. 3951-3964, 2017.

[30] K. Ma, W. Liu, K. Zhang, Z. Duanmu, Z. Wang, and W. Zuo, "End-to-end blind image quality assessment using deep neural networks," IEEE Transactions on Image Processing, vol. 27, no. 3, pp. 1202-1213, 2018.

[31] A. Mittal, A. K. Moorthy, and A. C. Bovik, "No-reference image quality assessment in the spatial domain," IEEE Transactions on Image Processing, vol. 21, no. 12, pp. 46954708, 2012.

[32] M. Oszust, "No-reference image quality assessment using image statistics and robust feature descriptors," IEEE Signal Processing Letters, vol. 24, no. 11, pp. 1656-1660, 2017.

[33] J. Wu, Z. Xia, H. Li, K. Sun, K. Gu, and H. Lu, "No-reference image quality assessment with center-surround based natural scene statistics," Multimedia Tools and Applications, vol. 77, pp. 20731-20751, 2017.

[34] Q. Jiang, W. Gao, S. Wang et al., "Blind image quality measurement by exploiting high-order statistics with deep dictionary encoding network," IEEE Transactions on Instrumentation and Measurement, vol. 69, no. 10, pp. 7398-7410, 2020.

[35] Q. Jiang, Z. Peng, S. Yang, and F. Shao, "Authentically distorted image quality assessment by learning from empirical score distributions," IEEE Signal Processing Letters, vol. 26, no. 12, pp. 1867-1871, 2019.

[36] A. Mittal, R. Soundararajan, and A. C. Bovik, "Making a "completely blind" image quality analyzer," IEEE Signal Processing Letters, vol. 20, no. 3, pp. 209-212, 2013.

[37] L. Zhang, L. Zhang, and A. C. Bovik, "A feature-enriched completely blind image quality evaluator," IEEE Transactions on Image Processing, vol. 24, no. 8, pp. 2579-2591, 2015.

[38] W. Xue, L. Zhang, and X. Mou, "Learning without human scores for blind image quality assessment," in Proceedings of the IEEE Conference on Computer Vision and Pattern Recognition, pp. 995-1002, IEEE, Portland, OR, USA, June 2013.

[39] L. Zhang, L. Zhang, X. Mou, and D. Zhang, "A feature similarity index for image quality assessment," IEEE Transactions on Image Processing, vol. 20, no. 8, pp. 2378-2386, 2011.

[40] Q. Wu, Z. Wang, and H. Li, "A highly efficient method for blind image quality assessment," in Proceedings of the 2015 IEEE International Conference on Image Processing (ICIP), pp. 339-343, IEEE, Quebec, Canada, September 2015.

[41] T. Ojala, M. Pietikainen, and T. Maenpaa, "Multiresolution gray-scale and rotation invariant texture classification with local binary patterns," IEEE Transactions on Pattern Analysis and Machine Intelligence, vol. 24, no. 7, pp. 971-987, 2002.

[42] Q. Wu, H. Li, F. Meng et al., "Blind image quality assessment based on multichannel feature fusion and label transfer," IEEE Transactions on Circuits and Systems for Video Technology, vol. 26, no. 3, pp. 425-440, 2016. 
[43] J. Gu, G. Meng, J. A. Redi, S. Xiang, and C. Pan, "Blind image quality assessment via vector regression and object oriented pooling," IEEE Transactions on Multimedia, vol. 20, no. 5, pp. 1140-1153, 2018.

[44] P. G. Freitas, W. Y. L. Akamine, and M. C. Q. Farias, "Noreference image quality assessment using orthogonal color planes patterns," IEEE Transactions on Multimedia, vol. 20, no. 12, pp. 3353-3360, 2018.

[45] Y. LeCun, Y. Bengio, and G. Hinton, "Deep learning," Nature, vol. 521, no. 7553, pp. 436-444, 2015.

[46] L. Zhang, L. Zhang, X. Liu, Y. Shen, and D. Wang, "Image exposure assessment: a benchmark and a deep convolutional neural networks based model," in Proceedings of the IEEE International Conference on Multimedia and Expo, San Diego, CA, USA, July 2018.

[47] A. Gaidon, Q. Wang, Y. Cabon, and E. Vig, "Virtual worlds as proxy for multi-object tracking analysis," in Proceedings of the IEEE Conference on Computer Vision and Pattern Recognition, pp. 4340-4349, IEEE, Las Vegas, NV, USA, June 2016.

[48] G. Ros, L. Sellart, J. Materzynska, D. Vazquez, and A. M. Lopez, "The synthia dataset: a large collection of synthetic images for semantic segmentation of urban scenes," in Proceedings of the IEEE Conference on Computer Vision and Pattern Recognition, pp. 3234-3243, IEEE, Las Vegas, NV, USA, June 2016.

[49] N. Mayer, E. Ilg, P. Häusser et al., "A large dataset to train convolutional networks for disparity, optical flow, and scene flow estimation," in Proceedings of the IEEE Conference on Computer Vision and Pattern Recognition, pp. 4040-4048, IEEE, Las Vegas, NV, USA, June 2016.

[50] ITU, BT. Itu-R. Methodology for the Subjective Assessment of the Quality of Television Pictures, ITU, Geneva, Switzerland, 2005.

[51] Z. Ni, L. Ma, H. Zeng, J. Chen, C. Cai, and K.-K. Ma, "ESIM: edge similarity for screen content image quality assessment," IEEE Transactions on Image Processing, vol. 26, no. 10, pp. 4818-4831, 2017.

[52] P. E. Debevec and J. Malik, "Recovering high dynamic range radiance maps from photographs," in Proceedings of the ACM Siggraph, pp. 369-378, Los Angeles, CA, USA, August 1997.

[53] Y. LeCun, B. Boser, J. S. Denker et al., "Backpropagation applied to handwritten zip code recognition," Neural Computation, vol. 1, no. 4, pp. 541-551, 1989.

[54] A. Krizhevsky, I. Sutskever, and G. E. Hinton, "ImageNet classification with deep convolutional neural networks," Advances in Neural Information Processing Systems, vol. 25, no. 2, pp. 1097-1105, 2012.

[55] K. Simonyan and A. Zisserman, "Very deep convolutional networks for large-scale image recognition," in Proceedings of the 2015 3rd IAPR Asian Conference on Pattern Recognition $(A C P R)$, IEEE, Kuala Lumpur, Malaysia, November 2015.

[56] C. Szegedy, W. Liu, Y. Jia et al., "Going deeper with convolutions," in Proceedings of the 2015 IEEE Conference on Computer Vision and Pattern Recognition (CVPR), IEEE, Boston, MA, USA, June 2015.

[57] K. He, X. Zhang, S. Ren, and J. Sun, "Deep residual learning for image recognition," in Proceedings of the IEEE Conference on Computer Vision and Pattern Recognition, pp. 770-778, IEEE, Las Vegas, NV, USA, June 2016.

[58] G. Huang, Z. Liu, L. V. D. Maaten, and K. Q. Weinberger, "Densely connected convolutional networks," in Proceedings of the IEEE Conference on Computer Vision and Pattern Recognition, pp. 2261-2269, IEEE, Honolulu, HI, USA, July 2017.
[59] X. Zhang, X. Zhou, M. Lin, and J. Sun, "ShuffleNet: an extremely efficient convolutional neural network for mobile devices," 2017, https://arxiv.org/abs/1707.01083.

[60] O. Russakovsky, J. Deng, H. Su et al., "Imagenet large scale visual recognition challenge," International Journal of Computer Vision, vol. 115, no. 3, pp. 211-252, 2015.

[61] M. Abadi, P. Barham, J. Chen et al., "TensorFlow: a system for large-scale machine learning," 2016, https://arxiv.org/abs/ 1605.08695 .

[62] D. Kingma and J. Ba, "Adam: a method for stochastic optimization," in Proceedings of the International Conference on Learning Representations, San Diego, CA, USA, May 2015.

[63] S. Su, Q. Yan, Y. Zhu et al., "Blindly assess image quality in the wild guided by a self-adaptive hyper network," in Proceedings of the 2020 IEEE/CVF Conference on Computer Vision and Pattern Recognition (CVPR), pp. 3667-3676, IEEE, Seattle, WA, USA, June 2020.

[64] Z. Ni, H. Zeng, L. Ma, J. Hou, J. Chen, and K.-K. Ma, "A gabor feature-based quality assessment model for the screen content images," IEEE Transactions on Image Processing, vol. 27, no. 9, pp. 4516-4528, 2018.

[65] W. Xue, L. Zhang, X. Mou, and A. C. Bovik, "Gradient magnitude similarity deviation: a highly efficient perceptual image quality index," IEEE Transactions on Image Processing, vol. 23, no. 2, pp. 684-695, 2014. 